\title{
Ruptured Abdominal Aortic Aneurysm Misdiagnosed as Acute Appendicitis
}

\author{
Hassan Adnan Bukhari, Mohammad Amin Mirza \\ Department of Surgery, Umm Al-Qura University, Makkah, Saudi Arabia.
}

\section{Corresponding Author:}

Dr. Hassan Adnan Bukhari

Email: drhb2000@yahoo.com

This is an Open Access article distributed under the terms of the Creative Commons Attribution License (creativecommons.org licenses/by/3.0).

Received

Accepted

February 15, 2017

Published

June 21, 2017

August 5, 2017

\begin{abstract}
Background: Ruptured abdominal aortic aneurysm is a serious life-threatening condition, which is usually prevalent in old age group. Unusual presentation in young patient is very difficult to diagnose and necessitates high index of suspicion. Any deviation from the normal presentation of acute appendicitis mandates further investigation. Case Report: This case report presents a very rare cause of abdominal pain in young patient that mimic acute appendicitis. The patient had abdominal CT scan, which revealed extensive abdominal aortic aneurysm with impending rupture. He was immediately transferred to tertiary hospital to be evaluated and managed by vascular surgeon. Emergent surgery was performed with uneventful recovery. Conclusion: Acute appendicitis is the most common cause of abdominal pain in young patient. However, treating surgeon should have high index of suspicious for any other causes the mimic acute appendicitis and could carry very high morbidity and mortality if not diagnosed early.
\end{abstract}

Keywords: Appendicitis, Aortic Aneurysm, Abdominal Pain, Vascular Surgical Procedures.

\section{Introduction}

Ruptured abdominal aortic aneurysm (RAAA) is a life threatening emergency [1]. The clinical presentation of RAAA ranges from asymptomatic up to sudden death [1]. Abdominal aortic aneurysm (AAA) is more common in elderly patients, which occur secondary to atherosclerotic changes and intimal injury superimposed by hypertension [2]. The emergency presentation is usually acute central abdominal pain and picture of hemorrhagic shock [3]. RAAA in young patients is very rare and it is usually due to developmental or genetic derangement like connective tissue disorders.

Diagnosis of acute appendicitis depends on clinical suspicion more than laboratory or imaging finding [4]. Literature review of published case reports shows that RAAA in young patients is very rare to be misdiagnosed as acute appendicitis because it is usually not suspected $[5,6]$. Pain in the right lower quadrant raises the suspicion of appendicitis but not a pathognomonic sign.

\section{Case Report}

A 29-year-old male healthy patient presented to emergency department (ED) complaining of abdominal pain for one day. The pain was sudden in onset, constant, non-radiating and localized to right lower quadrant. It was associated with two episodes of vomiting but there were no other gastrointestinal manifestations. Review of system was unremarkable. He denied any similar attacks in the past and his past medical/surgical history and family history were not significant. On physical examination, the patient was conscious and oriented with no signs of respiratory distress. However, he was hypotensive and tachycardic. He responded to fluid challenge and remained stable. Abdominal examination revealed localized tenderness in the right lower quadrant with positive rebound tenderness and Rovsing's sign. Rest of examination was unremarkable. Laboratory investigations showed mild leukocytosis and anemia with mild renal impairment. The rest was within normal range. Based on clinical examination and laboratory test, 
the diagnosis of acute appendicitis was suspected and the patient was admitted, rehydrated and given intravenous antibiotics to prepare him for laparoscopic appendectomy. The patient never looked toxic despite one episode of hypotension and tachycardia on presentation. His abdominal examination did not deteriorate despite passing almost 24 hours since admission. Therefore, abdominal ultrasonography was obtained, which only showed small-uncomplicated left ureteric stone and did not visualize the appendix. Decision of abdominal imaging with computed tomography (CT) without contrast was performed to reach a proper diagnosis. Surprisingly, CT scan showed large abdominal aortic aneurysm extending one $\mathrm{cm}$ below origins of renal arteries down to iliac arteries with a maximum diameter $6.5 \mathrm{~cm}$ [Fig.1,2]. Immediately, he was transferred to another hospital for vascular intervention. The patient underwent open AAA repair with interposition graft. Recovery was uneventful but he developed large incisional hernia. Patient and his family were counseled for genetic testing to identify the genetic cause of this early AAA.

\section{Discussion}

Abdominal aortic aneurysm (AAA) is defined as pathological local dilatation of abdominal aorta more than 50\%. Ruptured abdominal aortic aneurysm (RAAA) is a serious emergency condition requiring immediate intervention. It is usually a disease of elderly and found in $1 \%$ of all patients over the age of 50 with a mortality rate that can reach $50 \%$ [7] and up to $80 \%$ if ruptured [8]. Acute appendicitis is the most common cause of abdominal pain in young male patients. Presentation of RAAA in young patient could be very challenging and needs high index of suspicion; otherwise delayed diagnosis and intervention will carry very high morbidity and mortality. AAA is categorized based on etiology. Acquired AAA is more common in adult age group and congenital AAA is more in pediatrics. Acquired AAA develops secondary to atherosclerotic changes and

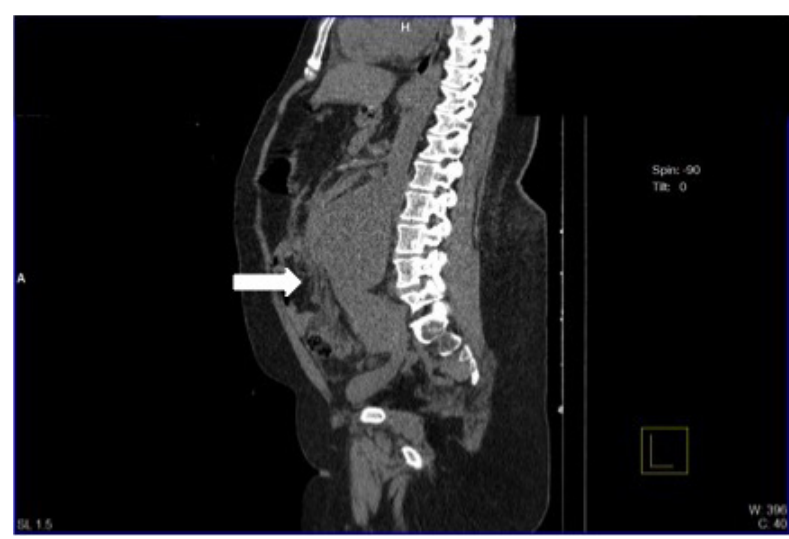

Fig.1: Plain abdominal CT scan (sagittal view) without contrast shows large dissecting abdominal aortic aneurysm extending into both iliac arteries with large retroperitoneal hematoma (arrow).

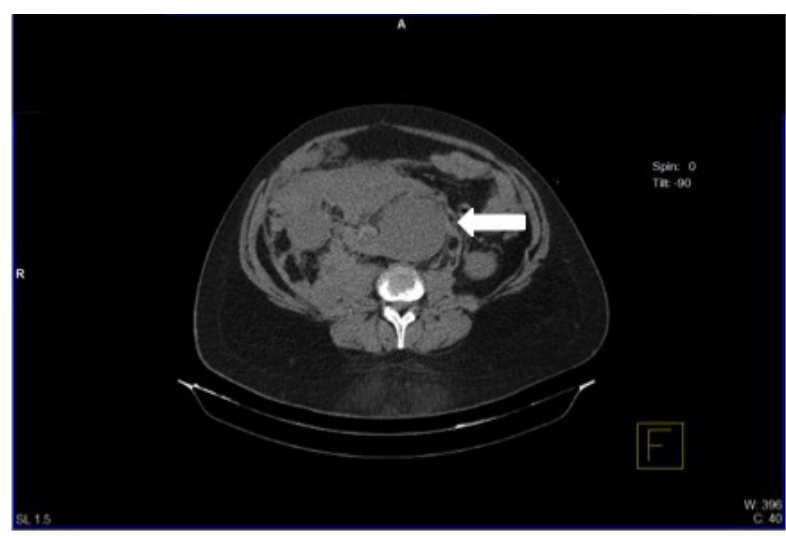

Fig.2: Plain abdominal CT scan without contrast shows large dissecting abdominal aortic aneurysm starting inferior to renal arteries with large retroperitoneal hematoma (arrow).

intimal injury superimposed by hypertension. On the other hand, congenital AAA is formed due to localized truncular form of arterial malformation secondary to the arrest of developmental formation during embryogenesis [6]. Congenital AAA is a rare and poorly understood entity. Upto date, fewer than thirty cases of pediatric AAA has been published but none of them presented with rupture [6]. Congenital AAA usually presents with symptomatic pulsatile abdominal mass and less commonly with reno-vascular hypertension and renal failure. Prompt intervention is needed to avoid disastrous outcome. Treatment of RAAA in young patients is guided by adult literature. There are two 
treatment modalities: open repair and endovascular aneurysm repair (EVAR) [6]. During open repair, native tissue can be used in pediatric patients and synthetic graft for adult. For RAAA in unstable patient, open repair with synthetic graft could be the reasonable option in young patients, which was performed for this case. This patient did not have any clinical feature of well-known connective tissue disorders indicating the possibility of congenital or genetic disease. Also he did not have family history of similar disease. In this case, a clinical picture of acute appendicitis was misleading and could have obscured the real diagnosis of a high-mortality condition like RAAA.

\section{Conclusion}

The treating surgeon should have high index of suspicion for other causes of abdominal pain in young patient especially when patient's presentation deviates from classical presentation. Initially, this patient was hypotensive on presentation, which is not typical for uncomplicated acute appendicitis. Those patients need further investigation to reach a proper diagnosis in a timely fashion to prevent morbidity and mortality.

Acknowledgement: Dr. Nabil Awad, medical intern for collecting the CT scan films and arranging the file.

Contributors: MAM: manuscript editing, critical inputs into the manuscript; HAB: manuscript drafting, and collection of patient details. HAB will act as guarantor. Both authors approved the final version of the manuscript.

Funding: None; Competing interests: None stated.

\section{References}

1. Schmitz-Rixen T, Keese M, Hakimi M, Peters A, Böckler D, Nelson K, et al. Ruptured abdominal aortic aneurysm - epidemiology, predisposing factors, and biology. Langenbeck's Arch Surg. 2016;401:275-288.

2. Khashram M, Williman JA, Hider PN, Jones GT, Roake JA. Systematic review and meta-analysis of factors influencing survival following abdominal aortic aneurysm repair. Eur J Vasc Endovasc Surg. 2016;51:203-215.

3. Dumont F, Goere D, Honore C, Elias D. Abdominal surgical emergencies in patients with advanced cancer. J Visc Surg. 2015;152(6 Suppl): S91-S96.

4. Hines JJ, Paek GK, Lee P, Wu L, Katz DS. Beyond appendicitis; radiologic review of unusual and rare pathology of the appendix. Abdom Radiol. 2016;41:568581.

5. Sterpetti AV, Hunter WJ, Schultz RD, Congenital abdominal aortic aneurysms in the young. Case report and review of the literature. J Vasc Surg. 1988;7:763769.

6. Wang Y, Tao Y. Diagnosis and treatment of congenital abdominal aortic aneurysm: a systematic review of reported cases. OJRD 2015;1.

7. Vu KN, Kaitoukov Y, Morin-Roy F, Kauffmann C, Giroux MF, Therasse E, et al. Rupture signs on computed tomography, treatment, and outcome of abdominal aortic aneurysms. Insights Imaging. 2014;5:281-293.

8. Li X, Zhao G, Zhang J, Duan Z, Xin S. Prevalence and trends of the abdominal aortic aneurysms epidemic in general population - A meta-analysis. PloS One. 2013;8:e81260. 